\title{
Guided $C^{2}$ Spline Surfaces with V-shaped tessellation
}

\author{
K. Karčiauskas and J. Peters
}

Dept. of Mathematics and Informatics, Vilnius University and Dept. CISE, University of Florida

\begin{abstract}
The guided spline approach to surface construction separates surface design and surface representation by constructing local guide surfaces and sampling these by splines of moderate degree. This paper explains a construction based on tessellating the domain into $\mathrm{V}$-shaped regions so that the resulting $C^{2}$ surfaces have $G^{2}$ transitions across the boundaries of the V-shapes and consist of tensor-product splines of degree $(6,6)$ with patches of degree $(4,4)$ forming a central cap.
\end{abstract}

\section{Introduction}

Our goal is to smoothly fill or cap a multi-sided hole in a $C^{2}$ spline complex by a (small) finite number of spline patches and without introducing shape artifacts such as curvature fluctuation or loss of convexity. The surface cap will interpolate position, first and second derivative for spline boundary data up to degree 6 . This degree permits exact blends not only in the common setting of bicubic patch complexes, but also when joining features like cylinders and tori (see Figure 12). We follow the general strategy laid out and illustrated in [1]: we separate surface design and surface representation by constructing local guide surfaces and sampling these by splines of moderate degree. For a guide surface $\mathrm{g}: \mathbb{R}^{2} \rightarrow \mathbb{R}^{3}$, a tessellation map $\tilde{\rho}: \mathbb{R}^{2} \rightarrow \mathbb{R}^{2}$, the sampling
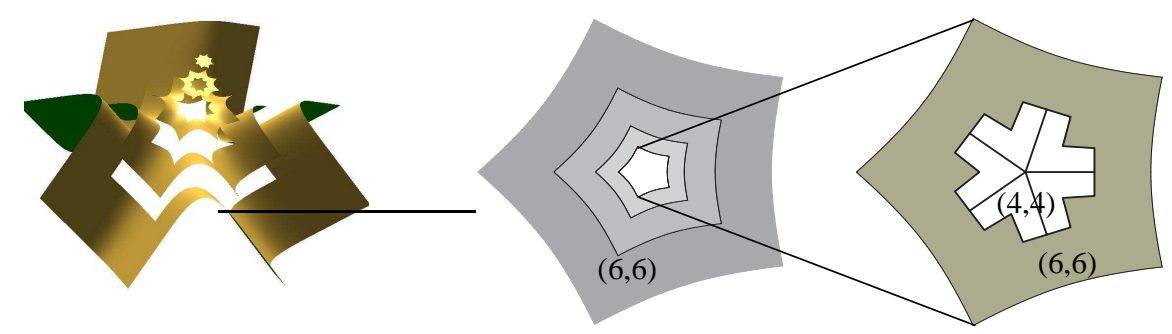

Fig. 1. (left) Sequence of guided surface rings and cap in $\mathbb{R}^{3}$, offset to show the structure. (middle) Surface rings of degree $(6,6)$ constructed according to [2]. (right) The focus of this paper: transition ring of degree $(6,6)$ and final cap consisting of $n$ V-shaped surface pieces of degree $(4,4)$.

operator $H: \mathbf{g} \circ \tilde{\rho} \mapsto \mathbf{x}$ maps the composition of the guide and the tessellation map to the final surface representation $\mathbf{x}:=H(\mathbf{g} \circ \tilde{\rho})$. We can interpret this as $H$ deciding 
the formal continuity and $\mathbf{g}$ expressing the design intent. In particular, $\mathrm{g}$ is a surface piece that outlines the intended shape, but need not fit the requirements of a typical modeling pipeline due to its representation or lack of continuity with the existing data. $H$ takes data from $\mathbf{g}$ to approximately rebuild $\mathbf{g}$ but in a framework suitable for standard design cycles. The basic construction options for $H$ and $\tilde{\rho}$ have been laid out in detail in $[2,1]$. The remaining, nontrivial technical challenge in devising surface caps is to find good transitions $\tau: \mathbb{R}^{2} \rightarrow \mathbb{R}^{2}$ between maps $\rho$ and $\sigma$, where $\rho: \mathbb{R}^{2} \rightarrow \mathbb{R}^{2}$ is the parameterization of the surrounding data and $\sigma: \mathbb{R}^{2} \rightarrow \mathbb{R}^{2}$ tessellates the immediate neighborhood of the pre-image of a central point (see Figure 2, dark, blue areas).
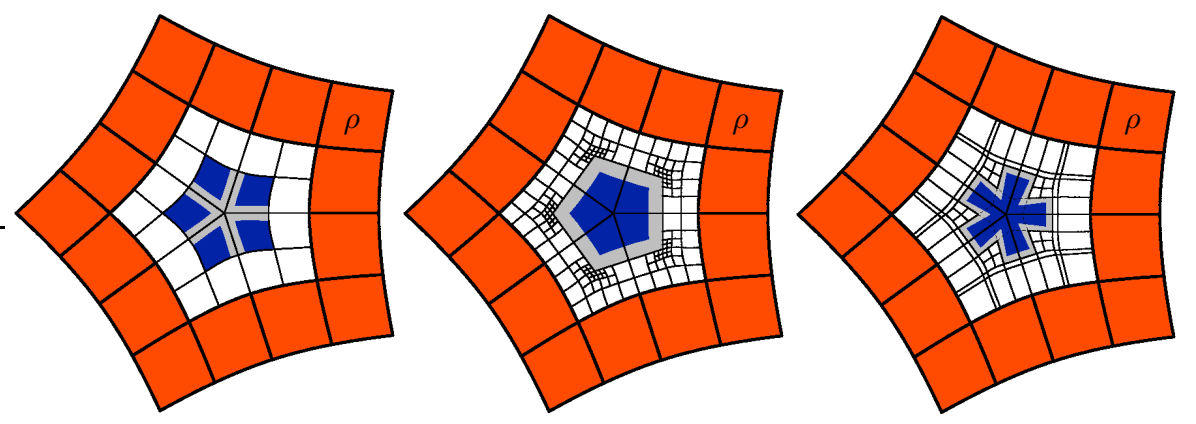

Fig. 2. Three different tessellations used respectively for surface constructions of degree $(8,8)$ [1], $(6,6)$ [3] and $(6,6)$ [this paper]. The $3 n$ quadrilaterals (quads) of the outermost (orange) rings are parametrized by maps $\rho$ of degree $(3,3)$, for example the characteristic map of CatmullClark subdivision. The innermost (blue) quads are parametrized by a map $\sigma$, or, alternatively, (middle and right) by a bilinear map $\beta$. The transition regions are parametrized by maps $\tau$. The edges with grey underlay indicate the technically most interesting transitions, the $G^{2}$ transitions. (left) Tessellation according to [1]: $\operatorname{deg}(\sigma)=\operatorname{deg}(\tau)=(5,5)$. (middle) Tessellation according to [3]: $\operatorname{deg}(\sigma)=(3,3), \operatorname{deg}(\beta)=(1,1)$ and $\operatorname{deg}(\tau)=(4,4)$. (right) Tessellation according to this paper: $\operatorname{deg}(\sigma)=(3,3), \operatorname{deg}(\beta)=(1,1)$ and $\operatorname{deg}(\tau)=(4,4)$.

Two finite constructions of this type have been devised in [3,1]. In [1], (Figure 2, left), $\sigma$ and $\tau$ are of degree $(5,5)$ and the key to the construction is that the partial derivatives $\partial^{k} \sigma, k=0,1,2$, of $\sigma$ on the $n$ sector partitioning edges (cf. Figure 3, left) of the $n$-sided cap are of degree $2 k+1$. There are $3 n$ polynomial pieces in $\tau$. In [3], (Figure 2, middle), $\sigma$ and $\tau$ are of degree $(3,3)$ and $(4,4)$ respectively, and there are $30 n$ polynomial pieces in $\tau$. The key technical achievement of this complex construction is that the partial derivatives $\partial^{k} \sigma$ across the outer $n$-gon boundaries of $\sigma$ are of degree $k+1$. This yields patches of degree $(4,4)$ surrounding the central point. The present paper introduces a third parameterization, (Figure 2, right), closely related to second construction, but simpler and unusual in its layout (see Figure 1). It results in 16n polynomial pieces in $\tau$, such that the outer boundaries of $\sigma$ are of degree $k+1$. Again, this yields $C^{2}$ surfaces with splines of degree $(6,6)$ in the transition and patches of degree $(4,4)$ surrounding the central point. The unusual V-shaped layout is inherited from the tessellation of the neighborhood of the pre-image of the central point. This 
tessellation is described in Section 2. The tessellation needs to transition smoothly to a standard parameterization of surrounding data for the transition between central cap and guided approach to work. The transition tessellation is defined in Section 3. Section 4 then defines the surface cap. The advantage of the present construction

- compared to [1] is the lower overall degree,

- compared to [3] the halving of the number of patches, simpler layout and stability for higher valences.

\section{A V-shaped tessellation map}

The basic idea underlying the tessellation was introduced in [4] in order to generate $C^{2}$ surfaces of least polynomial degree. While the approach advanced our technical repertoire, the accompanying algorithm was of limited use due to bad geometric properties for valences above 6 . Specifically, the low degree was based on a parameterization of the unit $n$-gon and, for each corner, the independent local parameters $u$ and $v$ were associated with the directions of consecutive $n$-gon edges. Unfortunately, the angle between consecutive edges approaches $180^{\circ}$ as the valence increases, leading in the limit for $n \rightarrow \infty$ to singularity, and much earlier to self-intersections of the Bézier control structure associated with the prolongation $\tau_{e}$ of the parameterization across the vertices (see Figure 3, middle top). [3] proposed a solution to this degeneracy, based on the observation that a finer partition allows to locally resolve the near-singularity. However, the construction is complex and applies reasonably only up to $n=8$ (which, in practice, usually suffices).
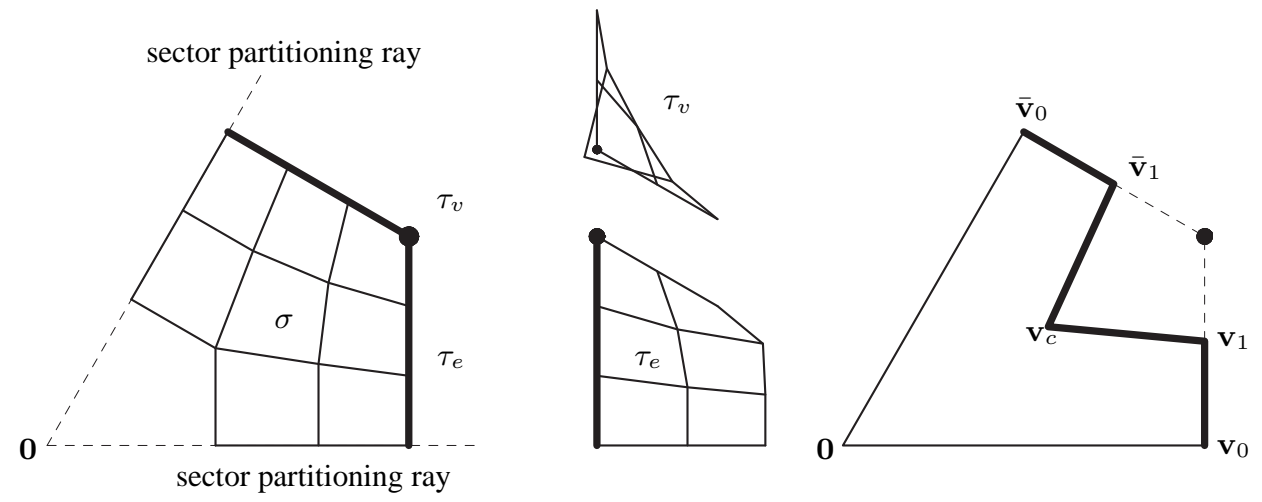

Fig. 3. One sector, valence $n=6$ : (left) The fat edges of $\sigma$ form a sector of a $n$-gon. (middle) The prolongations of $\sigma$ according to [4] (top) $\tau_{v}$ across the corner opposite the origin $\mathbf{0}$ and (bottom) $\tau_{e}$ across an edge. (right) New V-shaped domain after cutting out the obtuse angle of the $n$-gon.

Here, we propose a more efficient solution of this geometric problem keeping only the algebraic flavor of [4]. The idea is to cut out the obtuse angles and arrive at a 
$\mathrm{V}$-shaped domain for each sector of $\sigma$ as in Figure 3 right. We parametrize along $\mathbf{v}_{0}, \mathbf{v}_{1}, \mathbf{v}_{c}, \overline{\mathbf{v}}_{1}, \overline{\mathbf{v}}_{0}$ by $\sigma$ so that the boundary is piecewise linear, the first transversal derivative piecewise quadratic and only the second transversal derivative is of actual degree 3. Along the edge $\mathbf{v}_{0}, \mathbf{v}_{1}$ (Figure 4 left), the map consists of a single piece, along $\mathbf{v}_{1}, \mathbf{v}_{c}$ of two pieces to be able to create a consistent match at $\mathbf{v}_{c}$. The pieces are $C^{2}$ connected and, in particular, the pieces join correctly and are consistent at $\mathbf{v}_{c}$ (Figure 4 middle) and at the corners $\mathbf{v}_{1}$ (Figure 4 right).

The parameterization is chosen symmetric with respect to rotation by one sector. Each sector map $\sigma$ is symmetric with respect to the bisectrix of the sector. We abbreviate

$$
\alpha:=\frac{2 \pi}{n}, \mathrm{c}:=\cos \alpha, \mathrm{s}:=\sin \alpha, \mathbf{0}=(0,0), \hat{\mathrm{c}}:=\cos \frac{\alpha}{2}, \hat{\mathrm{s}}:=\sin \frac{\alpha}{2} .
$$

A sector is bounded by the sector partitioning rays $\overline{\mathbf{0 , ( 1 , 0 )}}$ and $\overline{\mathbf{0 , ( c , s )}}$ and the Vshaped domain is the polygon (cf. Figure 3 right)

$$
\left(\mathbf{0}, \mathbf{v}_{0}, \mathbf{v}_{1}, \mathbf{v}_{c}, \overline{\mathbf{v}}_{1}, \overline{\mathbf{v}}_{0}\right), \quad \mathbf{v}_{0}:=\left(z_{0}, 0\right), \mathbf{v}_{1}:=\left(z_{0}, z_{1}\right), \mathbf{v}_{c}:=\left(z_{2} \hat{\mathbf{c}}, z_{2} \hat{\mathbf{s}}\right),
$$

i.e. in terms of three parameters $z_{0}, z_{1}, z_{2}$. We split the V-shape into three quadrilaterals as shown in Figure 6, left.

Later on, we parameterize the $\mathrm{V}$-shape by three bilinear maps $\beta$, defined by the boundaries of the quadrilaterals. Here we focus on a higher-order parameterization
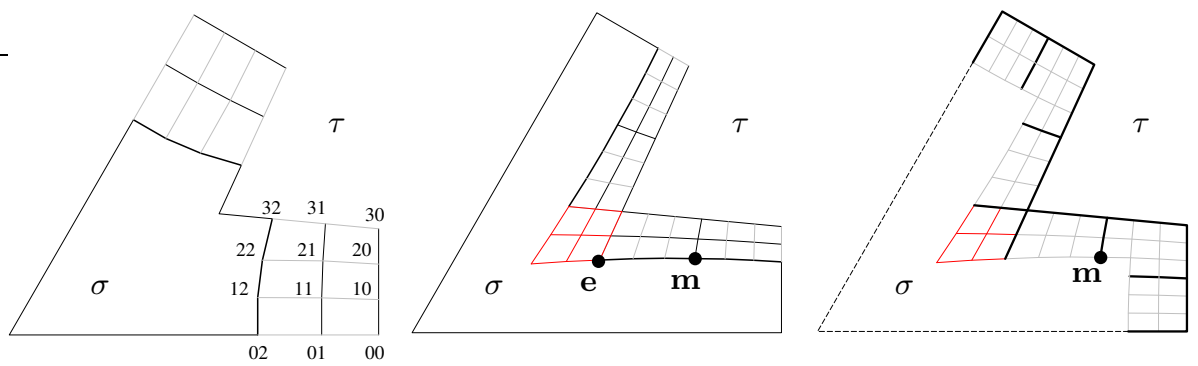

Fig. 4. (left) Coefficients of $\sigma$ at the tip of the V-shape. (middle) Two polynomial pieces of $\sigma$ along the edge $\mathbf{v}_{1} \mathbf{v}_{c}$ and their symmetric counterparts along $\overline{\mathbf{v}}_{1} \mathbf{v}_{c}$ join to form consistent $C^{2}$ data at $\mathbf{v}_{c}$. (right) An extra subdivision at the V-tips illustrates the uniform 'tensor-border' strip of the second derivatives prescribed by the tessellation $\sigma$.

$\sigma$ that plays a crucial role in the construction. We need only construct the derivatives of $\sigma$ up to second order, across the border $\left(\mathbf{v}_{0}, \mathbf{v}_{1}, \mathbf{v}_{c}, \overline{\mathbf{v}}_{1}, \overline{\mathbf{v}}_{0}\right)$ delineated as fat lines in Figure 3, right. These 'tensor-border' data will define the transition map $\tau$ along the outline of the V-shape, and, in turn, is used to compute the transition between the central cap and the guided rings.

The tensor-border data, position, first and second derivative, are defined by three layers of Bézier coefficients. The first layer is piecewise linear and the second piecewise quadratic as follows. 


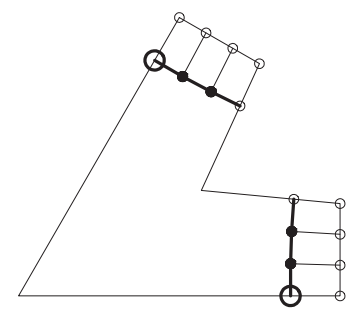

(a)

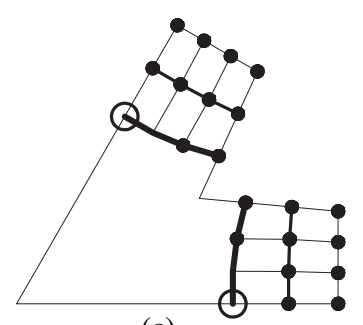

(c)

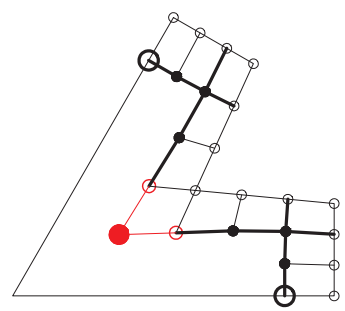

(b)

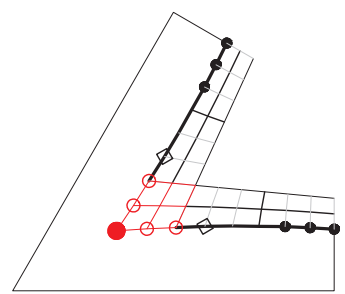

(d)

Fig. 5. Construction of $\sigma$. $\quad$ (a) and (b): quadratic first derivative layer: the two quadratic pieces are one subdivided quadratic. (a) The large circled point on sector partitioning ray is free. Small circles define linear maps in degree-raised form. (b) The Bézier coefficients of the $C^{1}$ extensions of the quadratic layers along $\mathbf{v}_{1}, \mathbf{v}_{c}$ and $\overline{\mathbf{v}}_{1}, \mathbf{v}_{c}$ coincide: the coefficient marked as a disk is on the bisectrix. This fixes the large-circled point in (a). (c) and (d): cubic second derivative layer. (c) Circled point of cubic layer on sector partitioning ray is free. (d) $C^{2}$ extensions of linear and quadratic layers define the points labeled $\circ$. The $\circ$ points and $C^{1}$ continuity define the points of cubic layers marked by diamonds. The diamonds, filled disks and $C^{2}$ continuity define the remaining Bézier points of the cubic layer. The Bézier points of the $C^{2}$ extensions of these cubic layers (along $\mathbf{v}_{1}, \mathbf{v}_{c}$ ) coincide: the coefficient marked as a disk is on the bisectrix. This fixes the large-circled point in (c).

(i) along the polygon edges $\left(\mathbf{0}, \mathbf{v}_{0}, \mathbf{v}_{1}, \mathbf{v}_{c}, \overline{\mathbf{v}}_{1}, \overline{\mathbf{v}}_{0}\right), \sigma$ is linear, and represented (thin lines in Figure 4, left) in degree-raised Bézier form:

$$
\sigma_{02}:=\left[\begin{array}{c}
x_{3} \\
0
\end{array}\right], \sigma_{01}:=\left[\begin{array}{c}
x_{1} \\
0
\end{array}\right], \sigma_{i 0}:=\left(1-\frac{i}{3}\right) \mathbf{v}_{0}+\frac{i}{3} \mathbf{v}_{1}, \sigma_{3 j}:=\left(1-\frac{j}{3}\right) \mathbf{v}_{1}+\frac{j}{3} \mathbf{v}_{c}
$$

with the prolongation $\mathbf{e}:=\frac{4}{3} \mathbf{v}_{c}-\frac{1}{3} \overline{\mathbf{v}}_{1}$.

(ii) The first, inner layer from the tip is quadratic in degree-raised form (medium lines in Figure 4, left). Since pieces corresponding to the same polygon edge join $C^{2}$, they are actually a single quadratic and

(iii) only the third layer (fat lines in Figure 4, left) is of actual degree 3 and consists of more than one polynomial piece per polygon edge.

Second and third layer are defined as

$$
\sigma_{11}:=\left[\begin{array}{l}
x_{1} \\
y_{1}
\end{array}\right], \sigma_{21}:=\left[\begin{array}{l}
x_{2} \\
y_{2}
\end{array}\right], \sigma_{12}:=\left[\begin{array}{l}
x_{3} \\
y_{3}
\end{array}\right], \sigma_{22}:=\left[\begin{array}{l}
x_{4} \\
y_{4}
\end{array}\right]
$$


(iv) We now subdivide $\sigma$ at the midpoints in the $u$ and the $v$ direction to obtain the layout of Figure $4, r i g h t$, and set $\mathbf{m}:=\left[\begin{array}{l}x_{5} \\ y_{5}\end{array}\right]$. By choosing

$$
\left(\begin{array}{l}
x_{1} \\
x_{2} \\
x_{3} \\
x_{4} \\
x_{5}
\end{array}\right):=\left(\begin{array}{ccc}
1 & \frac{-\hat{c}}{2 \hat{s}} & \frac{\hat{c}}{6} \\
\frac{8}{9} & \frac{-\hat{\imath}}{3 \hat{s}} & \frac{2 \hat{c}}{9} \\
\frac{13-c}{18} & \frac{(c-7) \hat{c}}{18 \hat{s}} & \frac{5 \hat{c}}{18} \\
\frac{5-c}{9} & \frac{(c-4) \hat{c}}{9 \hat{s}} & \frac{2 \hat{c}}{3} \\
\frac{189-19 c}{288} & \frac{(12 c-55) \hat{c}}{192 \hat{s}} & \frac{91 \hat{c}}{192}
\end{array}\right)\left(\begin{array}{c}
z_{0} \\
z_{1} \\
z_{2}
\end{array}\right),\left(\begin{array}{c}
y_{1} \\
y_{2} \\
y_{3} \\
y_{4} \\
y_{5}
\end{array}\right):=\left(\begin{array}{ccc}
0 & \frac{2}{9} & \frac{\hat{s}}{9} \\
0 & \frac{4}{9} & \frac{2 \hat{s}}{9} \\
\frac{-\mathrm{s}}{18} & \frac{c+2}{18} & \frac{\hat{s}}{3} \\
\frac{-s}{9} & \frac{c+2}{9} & \frac{2 \hat{s}}{3} \\
\frac{-19 \mathrm{~s}}{288} & \frac{9 c+47}{144} & \frac{17 \hat{s}}{36}
\end{array}\right)\left(\begin{array}{c}
z_{0} \\
z_{1} \\
z_{2}
\end{array}\right),
$$

we satisfy the $C^{2}$ continuity constraints. We summarize the result in Lemma 1.

Lemma 1. The tessellation map $\sigma$ defines $C^{2}$ data along $\left(\mathbf{v}_{0}, \mathbf{v}_{1}, \mathbf{v}_{c}, \overline{\mathbf{v}}_{1}, \overline{\mathbf{v}}_{0}\right)$. With $R$ the rotation by $\frac{2 \pi}{n}$, the maps of two adjacent sectors, $\sigma$ and $R \sigma$, join $C^{2}$ at $\overline{\mathbf{v}}_{0}$.

Using the explicit formulas, it is easy to formally verify Lemma 1. In particular, $C^{2}$ extensions of $\sigma$ on $\overline{\mathbf{v}_{0} \mathbf{v}_{1}}$ and $\overline{\overline{\mathbf{v}}_{0} \overline{\mathbf{v}}_{1}}$ join at the circled point in Figure 6,right. This will allow constructing $\tau$ of degree $(4,4)$. The intuition behind the approach and a specific, unique instance of $\sigma$ are sketched in Figure 5 .

\section{Transition $\tau$ from $\sigma$ to $\rho$}

We now build a transition $\tau$ between the innermost parameterization $\sigma$ of the $V$-shape and the outer parameterization $\rho$, the characteristic map of Catmull-Clark subdivision. One sector of the transition will consist of 16 patches of degree $(4,4)$ as shown in Figure 7. The Bézier net of one of the $n$ rotationally equivalent sectors of $\tau^{*}$, a precursor of $\tau$, is shown in Figure 6. The outer (dark grey) layers of Bézier control points are
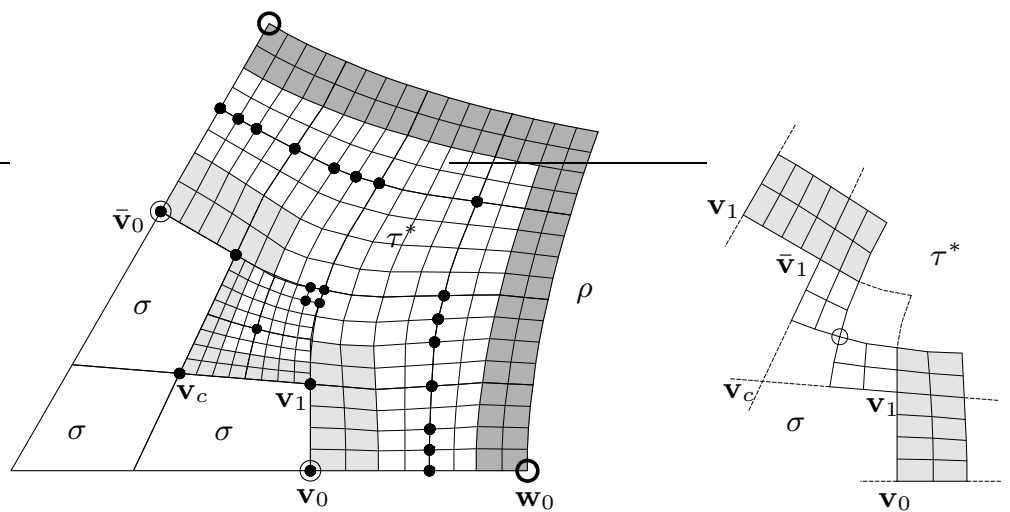

Fig. 6. (left) Structure of one sector of the tessellation $\tau^{*}$ between the origin and the outer tessellation $\rho$. (right) The transition $\tau^{*}$ near $\mathbf{v}_{c}$.

defined by the $C^{2}$ prolongation of $\rho$ and the inner (light grey) layers by $\sigma$. Then the internal $C^{2}$ constraints leave only the control points marked as black disks free to choose. 
Symmetry further cuts the number of free scalar values to 20 . For $n=3$, all 20 free parameters are determined using the functional $\mathcal{F}_{4}$ below. For $n>4$, we set $\mathbf{v}_{0}:=\lambda \mathbf{w}_{0}$, where $\lambda$ is subdominant eigenvalue of Catmull-Clark subdivision. The remaining 19 parameters are determined by minimizing the functional $\mathcal{F}_{4}$ summed over all 16 pieces of transition. Here $\mathcal{F}_{k}\left(f_{1}, f_{2}\right):=\mathcal{F}_{k}\left(f_{1}\right)+\mathcal{F}_{k}\left(f_{2}\right)$ and

$$
\mathcal{F}_{k}(f):=\int_{0}^{1} \int_{0}^{1} \sum_{i+j=k ; i, j \geq 0}\left(\begin{array}{l}
k \\
i
\end{array}\right)\left(\partial_{s}^{i} f \partial_{t}^{j} f\right)^{2} .
$$

Note that the functional is not applied to a surface in $\mathbb{R}^{3}$, but to a map in $\mathbb{R}^{2}$, and that these maps depend only on the valence, i.e. can be precomputed once and for all. Transition tessellations $\tau^{*}$ for various valences are shown in Figure 7. When compared
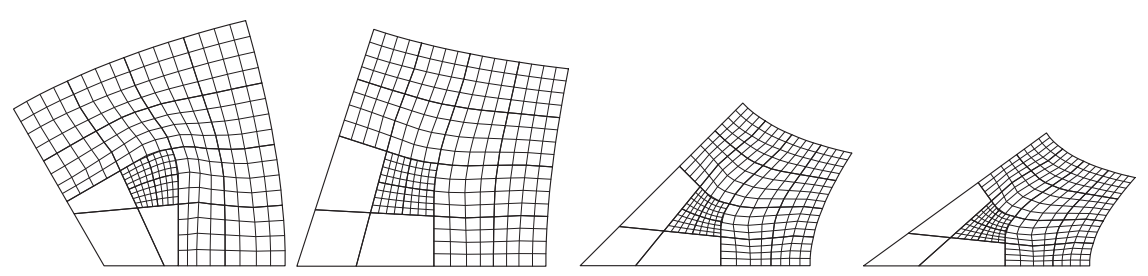

Fig. 7. Bézier net of the transition maps $\tau^{*}$. (from left to right): $n=3,5,8,10$. The case $n=6$ is shown in Figure 6 left.
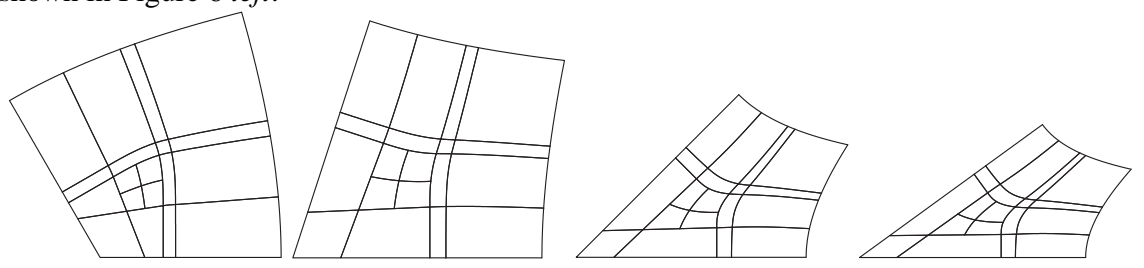

Fig. 8. Polynomial patch boundaries of the transition maps $\tau$ with adjusted spacing (from left to right): $n=3,5,8,10$.

to $\rho$, the first two derivatives of the transition maps $\tau^{*}$ in Figure 7 are one quarter the size. The univariate example in Figure 9, right, indicates that adjusting the spacing to make the derivatives larger improves the transition characteristics. Figure 8 shows the internal partition of the final map $\tau$ with the adjusted spacing according to Figure 10, right. Explicit formulas for the undetermined Bézier coefficients are provided in the Appendix. The underlying observation is that the change of lengths between $\tau^{*}$ of Figure 7 and $\rho$ causes problems when the guide is switched at the same time from $\mathrm{g}$ to $\mathbf{q}$ (see Figure 11 left). In contrast, unequal lengths within $\tau$ and correspondingly narrow patches sampling from one good guide $\mathbf{q}$ yields a stable procedure for all valences and does not appear to introduce any geometric artifacts (see Figure 11 right). 


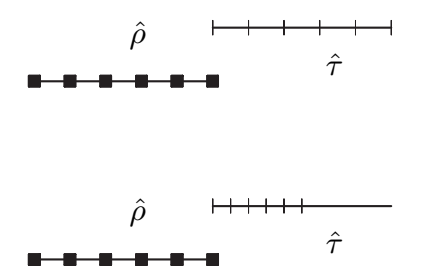

(a)

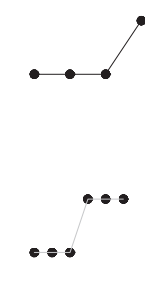

(b)

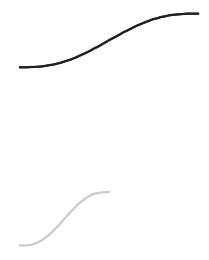

(c)

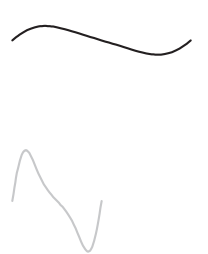

(d)

Fig. 9. Univariate example: transition between curves $\hat{\rho}$ and $\hat{\tau}$. (top) Long transition. (bottom) Short transition. (a) The black squares and partitions indicate two initial Bézier nets, here to be joined by a degree 5 transition. (b) shows the control net of the degree 5 curve (c) and (d) its curvature plot. The top transition would typically be preferred.
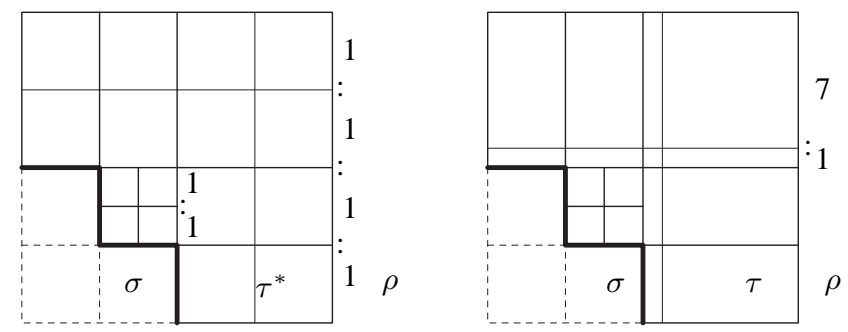

Fig. 10. (left) 1:1 spacing of the domain of the map $\tau^{*}$; (right) adjusted transition domain layout for the map $\tau$ (the image of the domain under $\tau$ was shown in Figure 8).

\section{Surface cap in $\mathbb{R}^{3}$}

The operator $H$ decouples the degree of the composition of guide and tessellation map from the degree of the resulting surface ring. However, for the cap, we need a lowdegree guide to arrive at a final surface that has low degree. After three guided surface rings, piecewise quartic guides do well since, in the $m$ th ring, the $k$ th order terms have decayed to $\lambda^{-k m}$ their original size, where $\lambda<1$ is the subdominant eigenvalue of Catmull-Clark subdivision. So we may replace any initial guide $\mathrm{g}$ by a $C^{2}$ piecewise polynomial guide $\mathbf{q}$ of total degree $d=4$.

Standard $C^{2}$ prolongation from the last guided ring attaches the transition surface $\mathbf{x}_{t}:=H(\mathbf{q} \circ \tau)$ to the surrounding surface; and $\mathbf{x}_{t}$ consists of 16 patches of degree $(6,6)$. Figure 11, left, illustrates that switching the guide from $\mathbf{g}$ to $\mathbf{q}$. Applying $\tau^{*}$ in the same, third surface ring has a detrimental effect on the curvature distribution. Figure 11 , right illustrates two alternative strategies that result in visually identical curvature images. The first switches to $\mathbf{q}$ in the fourth ring and uses $\tau^{*}$ in an additional fifth ring; the second switches guide and tessellation in the same, fourth ring, but uses $\tau$.

The resulting transition patchwork then smoothly joins the innermost cap. Since we only change the parameterization, not the image, the central V-shaped piece can alternatively be parametrized by $\sigma$ or by $\beta$ where $\beta$ consists of three bilinear pieces tracing out the $\mathrm{V}$-shape. For example, one piece has the coefficients $\mathbf{v}_{0}, \mathbf{v}_{1}, \mathbf{v}_{c}$ and a 

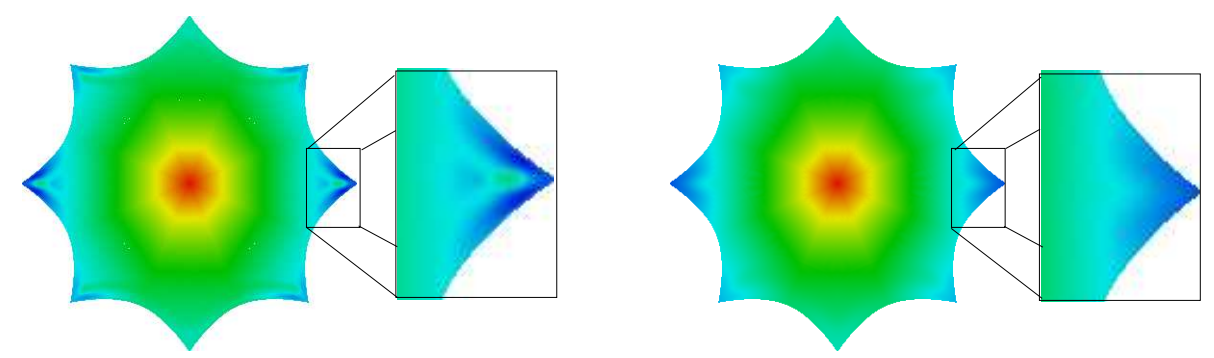

Fig. 11. (left) Switching both the tessellation map from $\rho$ to $\tau^{*}$ and the guide from $\mathbf{g}$ to $\mathbf{q}$ in the same ring yields an uneven Gauss curvature distribution (see enlargement). (right) Visually identical, better Gauss curvature distribution results from (i) switching to $\tau^{*}$ one ring later or (ii) switching in the same ring, but using the adjusted transition map $\tau$.

point on the edge $0 \overline{\mathbf{v}}_{0}$. That is, the central V-shaped piece of the surface can be defined by the composition $\mathbf{q} \circ \beta$ of degree $(4,4)$ i.e. be reinterpreted as a bilinearly trimmed piece of the guide $\mathbf{q}$.

Theorem 1 ( $C^{2}$ continuity). With $R$ the rotation by $2 \pi / n$ and $\beta$ a parameterization of the $V$-shaped domain consisting of three bilinear pieces,

(a) $\mathbf{q} \circ \beta$ and $\mathbf{q} \circ R \beta$ join $G^{2} \quad\left(G^{2}\right.$ capping $)$;

(b) $\mathbf{q} \circ \beta$ and $H(\mathbf{q} \circ \tau)$ join $G^{2} \quad\left(G^{2}\right.$ join to data $)$;

(c) $\operatorname{deg}(\mathbf{q} \circ \beta)=(4,4)$.

Proof. Since $\beta$ and $\sigma$ have the same $\mathrm{V}$-shaped range, the point sets $\mathbf{q} \circ \beta$ and $\mathbf{q} \circ$ $\sigma$ agree in $\mathbb{R}^{3}$ (the same piece of the $C^{2}$ guide surface is cut out). Since $\mathbf{q} \circ \sigma$ and $\mathbf{q} \circ R \sigma$ join $G^{2}$, (a) follows. For any preimage $E$ of a line segment of the boundary $\left(\mathbf{v}_{0}, \mathbf{v}_{1}, \mathbf{v}_{c}, \overline{\mathbf{v}}_{1}, \overline{\mathbf{v}}_{0}\right)$ of the V-shape, denote by $\operatorname{deg}_{E}(f)$ the degree of $f$ restricted to $E$. Then, by construction, $\operatorname{deg}_{E}\left(\partial^{j} \mathbf{q} \circ \sigma\right)=4+j$ for $j=0,1,2$. In particular, $\operatorname{deg}_{E}\left(\partial^{j} \mathbf{q} \circ\right.$ $\sigma) \leq 6$ and hence $H(\mathbf{q} \circ \tau)$ reproduces $\partial^{j}(\mathbf{q} \circ \sigma)$ along $E$. Therefore $\mathbf{q} \circ \sigma$ and $H(\mathbf{q} \circ \tau)$ join $C^{2}$. By the agreement of $\mathbf{q} \circ \sigma$ and $\mathbf{q} \circ \beta$ explained earlier, (b) holds. Since $\mathbf{q}$ is of degree 4 and $\beta$ of degree $(1,1)$, the composition $\mathbf{q} \circ \beta$ is of degree $(4,4)$.

Overall, from the outside towards the central point, the construction yields a sequence of surface rings $H(\mathrm{~g} \circ \rho)$ of $3 n$ patches of degree $(6,6)$, followed by a transition ring $H(\mathbf{q} \circ \tau)$ of 16 patches of degree $(6,6)$, followed by a cap $\mathbf{q} \circ \beta$ of $3 n$ patches of degree $(4,4)$.

\section{Discussion}

While a number of techniques now exist that automatically create curvature continuous blends [4-15], none fully meets the requirements of product-defining outer surfaces in high-end design of CAD/CAM applications. For example, [16] showed that shape deficiencies are intrinsic to all major subdivision surface constructions, no matter how 
carefully a designer might adjust the input data. Algorithms that build on, say CatmullClark, subdivision inherit the problems. Conversely, constructions that do not make use of multiple refinement steps and construct the blend from a minimal number of polynomial pieces typically pay for the abrupt transition of patch type with curvature fluctuations.

In contrast to the prevalent all-in-one approach, the guided approach explicitly separates the initial shape design from surface representation. The initial shape is defined via local surface fragments that need only obey few constraints. These fragments are then sampled (re-approximated) so that the output fits standard design cycles.

Compared to the $C^{2}$ scheme in [1], the presented approach has lower overall degree. Compared to [3] the number of patches is halved and the layout is simpler so that the scheme is stable even for higher valences. The curvature distribution of all three schemes is very similar.
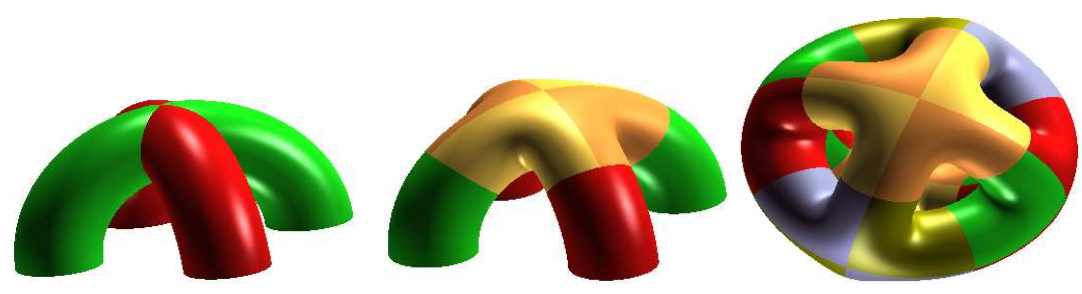

Fig. 12. (left and middle) Joining two torus pieces with curvature continuity. (right) Joining three tori.

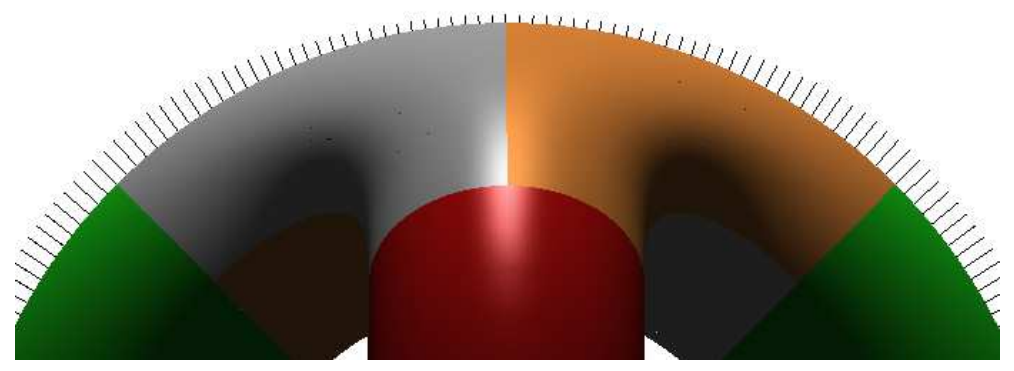

Fig. 13. Curvature Comb (normals scaled by Gauss curvature attached to surface points) for the three tori join.

Acknowledgment: This work was supported in part by grants DMI-0400214 and CCF-0430891.

\section{References}

1. Karčiauskas, K., Peters, J.: Guided $C^{2}$ spline surfaces. Computer Aided Geometric Design (2007) xx submitted. 
2. Karčiauskas, K., Peters, J.: Concentric tesselation maps and curvature continuous guided surfaces. Computer Aided Geometric Design 24(2) (2007) 99-111

3. Karčiauskas, K., Peters, J.: Parameterization transition for guided $c^{2}$ surfaces of low degree. In: Sixth AFA Conference on Curves and Surfaces Avignon, June 29-July 5, 2006. (2007) $\mathrm{xx}-10$ to appear.

4. Peters, J.: $C^{2}$ free-form surfaces of degree (3,5). Computer Aided Geometric Design 19(2) (2002) 113-126

5. Hahn, J.: Filling polygonal holes with rectangular patches. In: Theory and practice of geometric modeling (Blaubeuren, 1988). Springer, Berlin (1989) 81-91

6. Gregory, J.A., Hahn, J.M.: A $C^{2}$ polygonal surface patch. Comput. Aided Geom. Design 6(1) (1989) 69-75

7. Gregory, J.A., Zhou, J.: Irregular $C^{2}$ surface construction using bi-polynomial rectangular patches. Comput. Aided Geom. Design 16(5) (1999) 423-435

8. Ye, X.: Curvature continuous interpolation of curve meshes. Computer Aided Geometric Design 14(2) (1997) 169-190

9. Reif, U.: Analyse und Konstruktion von Subdivisionsalgorithmen für Freiformflächen beliebiger Topologie. Shaker Verlag, Aachen (1999)

10. Prautzsch, H.: Freeform splines. Comput. Aided Geom. Design 14(3) (1997) 201-206

11. Prautzsch, H., Umlauf, G.: Triangular $G^{2}$ splines. In P.J. Laurent, A. LeMéhauté, L.S., ed.: Curve and Surface Design, Vanderbilt University Press (2000) 335-342

12. Navau, J.C., Garcia, N.P.: Modeling surfaces from meshes of arbitrary topology. Comput. Aided Geom. Design 17(7) (2000) 643-671

13. Loop, C.: Second order smoothness over extraordinary vertices. In: Symp Geom Processing. (2004) 169-178

14. Ying, L., Zorin, D.: A simple manifold-based construction of surfaces of arbitrary smoothness. ACM TOG 23(3) (2004) 271-275

15. Levin, A.: Modified subdivision surfaces with continuous curvature. In: SIGGRAPH, ACM Transactions On Graphics. (2006) 1035-1040

16. Karciauskas, K., Peters, J., Reif, U.: Shape characterization of subdivision surfaces - case studies. Computer Aided Geometric Design 21(6) (2004) 601-614

\section{Appendix}

The transition map $\tau$ is defined by scalar values $\left[z_{0}, z_{2}, \ldots, z_{19}\right]$ (see Table 1 ) that define coefficients $\mathbf{p}_{1}, \ldots \mathbf{p}_{15}$ as indexed in Figure 14. The remaining coefficients are defined by symmetry and the $C^{2}$ conditions.

$$
\begin{aligned}
\mathbf{p}_{1} & :=\mathbf{v}_{0}:=\left(z_{0}, 0\right), \mathbf{p}_{2}:=\mathbf{v}_{1}:=\left(z_{0}, z_{1}\right), \mathbf{p}_{3}:=\mathbf{v}_{2}:=\left(z_{2} \hat{\mathbf{c}}, z_{2} \hat{\mathbf{s}}\right) \\
\mathbf{p}_{4} & :=\left(z_{3}, 0\right), \mathbf{p}_{5}:=\left(z_{3}, z_{4}\right), \mathbf{p}_{6}:=\left(z_{5}, 2 z_{4}\right), \mathbf{p}_{7}:=\left(z_{6}, z_{7}\right), \\
\mathbf{p}_{8} & :=\left(z_{8}, z_{9}\right), \mathbf{p}_{9}:=\left(z_{10}, z_{11}\right), \mathbf{p}_{10}:=\left(z_{12}, z_{13}\right), \mathbf{p}_{11}:=\left(z_{14} \hat{\mathrm{c}}, z_{14} \hat{\mathbf{s}}\right) \\
\mathbf{p}_{12} & :=\left(z_{15}, z_{16}\right), \mathbf{p}_{13}:=\left(z_{17} \hat{\mathrm{c}}, z_{17} \hat{\mathbf{s}}\right), \mathbf{p}_{14}:=\left(z_{18} \hat{\mathrm{c}}, z_{18} \hat{\mathbf{s}}\right), \mathbf{p}_{15}:=\left(z_{19} \hat{\mathrm{c}}, z_{19} \hat{\mathbf{s}}\right) .
\end{aligned}
$$




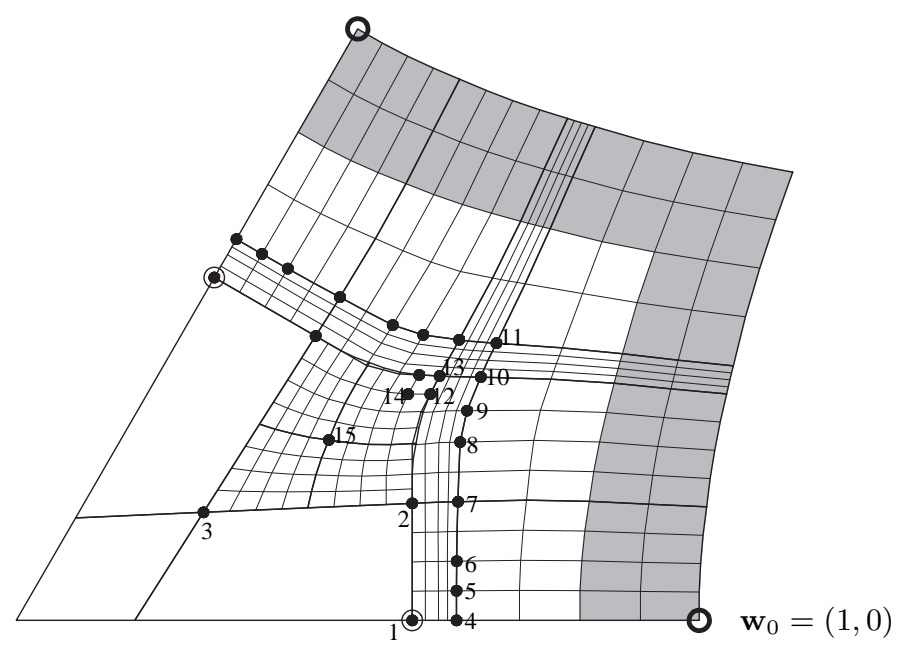

Fig. 14. Indices of the free points $\mathbf{p}_{i}$ of a half-segment.

Table 1. Scalar parameters defining $\tau$ for valence $n$.

\begin{tabular}{|c|c|c|c|c|c|c|c|c|c|}
\hline$i$ of $z_{i}$ & $n=3$ & $n=5$ & $n=6$ & $n=7$ & $n=8$ & $n=9$ & $n=10$ & $n=11$ & $n=12$ \\
\hline 0 & .34871 & .54999 & .57968 & .59851 & .61112 & .61994 & .62634 & .63113 & .63480 \\
1 & .28552 & .20389 & .17136 & .14718 & .12853 & .11373 & .10173 & .09183 & .08353 \\
2 & .29433 & .32590 & .31641 & .31374 & .31414 & .31610 & .31891 & .32217 & .32566 \\
3 & .41705 & .61441 & .64457 & .66326 & .67544 & .68368 & .68941 & .69349 & .69643 \\
4 & .07309 & .05140 & .04334 & .03727 & .03257 & .02882 & .02578 & .02326 & .02115 \\
5 & .41698 & .61450 & .64476 & .66354 & .67580 & .68412 & .68994 & .69409 & .69712 \\
6 & .41673 & .61563 & .64672 & .66613 & .67890 & .68767 & .69389 & .69841 & .70178 \\
7 & .29215 & .20589 & .17372 & .14945 & .13060 & .11559 & .10339 & .09331 & .08485 \\
8 & .41653 & .61752 & .64994 & .67032 & .68385 & .69324 & .70000 & .70500 & .70880 \\
9 & .43795 & .30923 & .26108 & .22468 & .19637 & .17382 & .15547 & .14030 & .12757 \\
10 & .40992 & .62393 & .65985 & .68238 & .69737 & .70781 & .71536 & .72099 & .72530 \\
11 & .50993 & .36235 & .30704 & .26515 & .23255 & .20656 & .18541 & .16790 & .15321 \\
12 & .39526 & .63705 & .68004 & .70687 & .72469 & .73712 & .74614 & .75288 & .75807 \\
13 & .58072 & .41754 & .35611 & .30937 & .27290 & .24378 & .22006 & .20040 & .18386 \\
14 & .75262 & .80556 & .81170 & .81568 & .81840 & .82035 & .82181 & .82294 & .82385 \\
15 & .33372 & .56708 & .60617 & .63077 & .64721 & .65873 & .66712 & .67342 & .67828 \\
16 & .53111 & .38919 & .33118 & .28738 & .25332 & .22617 & .20407 & .18576 & .17036 \\
17 & .65178 & .71175 & .71566 & .71856 & .72071 & .72233 & .72360 & .72462 & .72546 \\
18 & .60341 & .66137 & .66243 & .66379 & .66503 & .66607 & .66695 & .66769 & .66832 \\
19 & .45588 & .52668 & .52838 & .53095 & .53345 & .53570 & .53769 & .53946 & .54105 \\
\hline
\end{tabular}

